\title{
ЭФФЕКТИВНОСТЬ ЛЕЧЕНИЯ ПАЦИЕНТОВ С НЕАКТИВНОЙ СТАДИЕЙ ЭНДОКРИННОЙ ОФТАЛЬМОПАТИИ
}

\author{
Пантелеева О.Г., Батырбекова Ф.Х. \\ ФГБУ «НМИЦ ГБ им. Гельмгольца» Минздрава России, Москва
}

Направление тезиса 20. Междисциплинарный подход к лечению эндокринной офтальмопатии у пациентов с аутоиммунной патологией щитовидной железы.

Эндокринная офтальмопатия (ЭОП) - самостоятельное прогрессирующее заболевание органа зрения, ассоциированное с аутоиммунной патологией щитовидной железы (ЩЖ). Частота клинически выраженной ЭОП составляет 30-50\%. Если учитывать долю субклинических форм ЭОП, частота развития ЭОП достигает 70\% (Wei Y., Kang X..L и соавт, 2016). Выделяют активную и неактивную стадии ЭОП. Лечение активной стадии ЭОП не вызывает сомнений, однако вопрос необходимости лечения неактивной стадии дискутабелен.

ЦЕЛЬ: оценить эффективность лечения пациентов с неактивной стадией ЭОП.

МАТЕРИАЛЫ И МЕТОДЫ: В исследование включено 43 пациента (39 женщин, 4 мужчин) с неактивной стадией ЭОП, которые обследованы в течение 2019 года на базе ФГБУ «МНИЦ глазных болезней им.

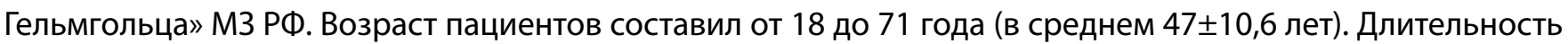
наблюдения - от 3 до 12 месяцев. До начала лечения и через 3-6 мес после лечения всем больным проводили стандартное офтальмологическое обследование, оценку цветового зрения, компьютерную периметрию (КП), компьютерную томографию (КТ) орбит с денситометрией мягких тканей орбиты. Оценку активности ЭОП проводили по шкале CAS (Clinical Active Score, 1989). Наличие оптической нейропатии (OH) подтверждали методами КП, изменениями цветового зрения и комплексом электрофизиологических исследований. У всех пациентов до начала лечения эндокринолог фиксировал лабораторный эутиреоз. Больным проводили комплексную медикаментозную терапию, физиотерапию в сочетании с локальным курсом глюкокортикоидной терапии.

PEЗУЛЬТАТЫ: в настоящее время Европейская группа по изучению эндокринной офтальмопатии (EUGOGO) рекомендует только топическую терапию с динамическим наблюдением пациентов с неактивной стадией ЭОП (Luigi Bartalena, Lelio Baldeschi, 2016), при этом не учитывают наличие признаков ОН. Проведенные нами инструментальные исследования доказали наличие латентной стадии ОН у всех больных, включенных в исследование. Объем медикаментозной терапии определяли выявленными причинами развития ОН. Физиотерапию (электрофорез в сочетании с магнитотерапией) назначали пациентам с признаками поражения ЭОМ (диплопия, положительный симптом Сикара, ретракция верхнего века). В процессе динамического наблюдения у 36 пациентов $(83,7 \%)$ отмечена положительная динамика в виде регрессии признаков ЭОП и улучшении зрительных функций, у 5 пациентов $(11,6 \%)$ регрессировали признаки ОН, но симптомы поражения ЭОМ сохранялись, у 2 пациента $(4,7 \%)$ отмечена отрицательная динамика, что мы связываем с нестабильным гормональным статусом в период наблюдения.

ВЫВОдЫ: все больные ЭОП, в т.ч. с неактивной стадией, требуют клинико-инструментального обследования офтальмолога. Выявление признаков ОН, даже ее латентной стадии, диктует необходимость проведения медикаментозной терапии по персонифицированной схеме, объем которой определяется причинами развития ОН. В 95,3\% наблюдений удалось добиться регрессии признаков ОН. Комбинация медикаментозной терапии с курсом физиотерапии позволила получить восстановление функций ЭОМ в 83,7\% наблюдений. Особое значение приобретает грамотное ведение пациента эндокринологом с целью стабилизации гормонального фона.

КЛЮЧЕВЫЕ СЛОВА: Эндокринная офтальмопатия, аутоиммунное заболевание, офтальмология, болезнь Грейвса. 